\title{
Robots for Real: Developing a Participatory Design Framework for Implementing Educational Robots in Real-World Learning Environments
}

\author{
Lykke Brogaard Bertel $^{1}$, Dorte Malig Rasmussen ${ }^{1}$, and Ellen Christiansen ${ }^{2}$ \\ ${ }^{1}$ Danish Technological Institute, Robot Technology, Odense, Denmark \\ $\{1 \mathrm{bb}, \mathrm{dmrn}\} @ \mathrm{dti} . \mathrm{dk}$ \\ ${ }^{2}$ Aalborg University, Department of Communication and Psychology, Aalborg, Denmark \\ ech@hum. aau. dk
}

\begin{abstract}
As educational service robots become increasingly accessible, the demand for methodologies that generate knowledge on r-learning applicable to real world learning environments equally increases. This paper proposes a participatory design framework for involving users in the development of robot-supported didactic designs and discusses its applicability to existing educational contexts on the basis of a case study on the implementation and use of the therapeutic robot seal Paro at a school for children with an autism diagnosis.
\end{abstract}

Keywords: Participatory design, Human-Robot Interaction, Education.

\section{Introduction}

For the educational robots of tomorrow to succeed as trainers, teachers and playmates, their appearance, interaction style and social behavior must match the users' needs and meet the demands of a changing educational context. This call for wellestablished guidelines to how such robots should be designed and in what ways interacting with them can support learning [1]. Thus new areas of research within Human-Robot Interaction (HRI) have emerged, focusing particularly on HRI in education, introducing terms such as r-learning [2] and Child-Robot Interaction [3]. Also the applicability of robots in specific educational curricula such as STEM teaching and language learning [4-6] and special needs education [7-10] has gained increased interest in recent years.

Robots for education are often categorized by their design and functionality [1112] e.g. as educational robotics (or hands-on robotics) as opposed to educational service robots (social, anthropomorphized robots) [2]. Since hands-on robotic kits generally have been argued to support a constructionist approach to learning [13] research within educational robotics has a tradition for including users in the design and development of such robots through participatory innovation processes [14-15].

C IFIP International Federation for Information Processing 2013 
Within educational service robots, on the other hand, a much clearer boundary is drawn between users and developers [2]. Users are usually not included in the design and development process and limited research has been conducted on the actual implementation of educational service robots in real-world learning environments [16]. Due to high technological complexity and cost combined with low system stability and usability, the majority of research on educational service robots is still based on highly controlled experiments in lab settings which cause certain limitations to the transferability and applicability of the results to real-world learning environments.

First of all, the majority of HRI studies use the Wizard of Oz method (WoZ), in which the robot is fully or partially tele-operated (often without the participants knowing) [17]. Besides the ethical implications WoZ may have (imagine a child revealing his deepest secrets to a robot pet, only to find it is being remotely operated by a researcher, teacher or perhaps parent), the constant demand for an operator reduces the usability of the robot and entails the risk of unrealistic expectations and thus disappointment and rejection of "real" (and perhaps less impressive) autonomous robots. Secondly, though the need for "long-term interaction" studies is often made explicit in calls for papers, the notion of "long-term" is still not really well-defined and the majority of these studies are characterized by what one might call "serial short-term interaction" in which for instance users participate in experiments once a week for a period of time [18]. Although these studies do explore users' ongoing interest in a robot when interaction is repeated, they do not take into account contextual factors such as the communication and motivational structures, social dynamics or cultural values in the learning environment that may also affect the interaction and user motivation. In addition, long-term r-learning studies are often conducted in dedicated "playtime" labs [19] overlooking the fact that children, and particularly children with special needs, need familiar, predictable environments to feel safe and can behave very differently in unfamiliar settings. Thirdly, many r-learning experiments are evaluated by quantitative metrics of measurements. This means, that statistical analysis is often applied to too small data sets [7] and that e.g. the number of dialogues between a child and a robot (e.g. [19]) is given priority rather than the quality of such dialogues. Finally, the advantage that experimental methods are said to have over field research when it comes to generalization induces a predominant preference for generalizable results; completely overlooking the importance of individualization as stressed in technology-enhanced learning and special needs education in particular.

To summarize, although r-learning experiments can indeed be of great relevance to the research community, knowledge produced within a theoretically construed setting will necessarily remain theoretical. Thus, a growing number of researchers argue that r-learning research, to have an impact on society, must be conducted in and in collaboration with society [16]. This paper is in line with this view and argues that a lot can be gained from the hands-on educational robotics' constructionist and participatory approach to didactics when exploring and developing educational service robots. Thus, the paper proposes a participatory design framework for developing robot-supported didactic designs in close collaboration with users and practitioners, and demonstrates its applicability to real-world learning environments in a case study on the implementation and use of the therapeutic robot seal Paro in autism education. 


\section{Time-Space-Structure: A Participatory Design Framework}

As users are increasingly viewed as active consumers of technology and co-producers of technological practice, new approaches to designing, developing and testing technology are also emerging, requesting systematic methods for including users' experiences, views and needs as resources and including users and practitioners as potential co-developers of future products and services. Participatory Design is particularly useful for this purpose [20] since it is indeed in line with the constructionist perspective, viewing people, social systems and technological development as interrelated, emphasizing the importance of stakeholder involvement and the value of concrete, context-dependent knowledge. This particular participatory design framework focuses on three components: Time, Space and Structure (TSS) as central to design processes with and for users and practitioners. These components are individually present in all user studies. However this paper proposes that particularly the balance between these components determines (1) the level of user participation and (2) the nature of the research questions that a particular user study can address.

\subsection{Time}

Time covers the length of the study in question. For studies addressing users' first impressions and initial attitudes towards educational service robots time is not necessarily a crucial component, although the often accompanied interest in attitude changes over time naturally increases the importance of extending the user study in time. The time invested is in itself a strategic choice, since a longer case study naturally requires more resources from both researchers and practitioners. At the same time, though, it allows for more research and development iterations as well as ongoing adjustment of the project according to newly gained knowledge and experiences and thus more reliable and replicable results. Our case study extended over a period of 3 months, which allowed users and practitioners to become familiar with the technology and to a certain extent include it as part of their everyday lives and practice.

\subsection{Space}

Space covers the place in which the study is conducted (e.g. field research as opposed to lab experiments) but do also contain several symbolic meanings. Firstly, a certain stability of the technology must be ensured before sending it out into 'space'. Early educational service robot prototypes are not well-suited for these studies unless developers are present at all times, which in turn require extensive resources. Secondly, researchers must ensure a "creative space", making room for ideas and views of users and practitioners. Participatory design is to a certain extent democratic, providing the opportunity for explorative and creative processes rather than dictating the use of the technology [20]. Finally, a "safe space" must be provided for participants to disclose their personal views and experiences, without the fear of being exposed, since dissenting views and opinions are also essential and enable the discovery of new aspects of the implementation and interaction with the technology. 


\subsection{Structure}

Structure covers the level of control in the study. In experiments control is crucial to ensure validity and generalizable results. In participatory design processes, however, too much control can demotivate the participants or even induce reluctance towards the researchers or the technology. On the other hand a total lack of control could possibly leave the development unfocused, participants insecure and unmotivated, and the technology unused. In order to provide the best conditions for creative processes, we argue the necessity of a structured but flexible framework for participants to navigate. In our case study this was done by devising a blueprint for the process in collaboration with the participants, by planning workshops to collect and share experiences as well as by introducing goal-setting as a way to facilitate direction and motivation.

\section{TSS in Practice: A Case Study}

To investigate the applicability of TSS in practice we conducted a three month case study on the use of the therapeutic robot seal Paro at a school for children with autism. The purpose of the case study was two-fold; to explore the potential of robots to promote motivation, communication, play and learning in autism education, to investigate the importance of structure in the TSS-framework as well as to identify additional contextual factors that both influence and are influenced by the technological intervention. A total of three Paros were implemented in 3 existing teaching teams of 45 teachers and 6-8 students each. At the school the teaching teams are divided by age: T1 (6 children aged 6-9), T2 (7 children aged 10-13) and T3 (8 children aged 14-17) although the students are all at a similar stage of cognitive development (approx. 0-1 years of age) in which most have no spoken language and use alternative tools for communication. To evaluate the importance of structure in the TSS-framework the groups were differentiated by the stage in which goal-setting activities were initiated and thus the level of structure (ranging from structured, to semi-structured and nonstructured). The research setup comprised the following three phases:

1. A 3 week exploration phase initiated the study. In this phase the Paros were introduced and made available to the teachers without any limitations or restrictions of use. The robots were explored with all students in different setups (i.e. individually, in pairs and in groups) with different levels of teacher involvement (independent, guided or prompted interactions). During this phase we were present most of the time, mainly to ensure no technical issues were obstructing the introduction as well as to gain knowledge on the context and to create a baseline on the basis of the student's immediate responses to Paro. Data collection methods consisted of participating and non-participating observations, in situ interviews and video material. The teachers in T3 defined the goals for the use of Paro in their team early at this stage, i.e. facilitating social interaction and supporting the transition from familiar to unfamiliar settings (e.g. going somewhere new, meeting new people etc.)

2. A 3 week co-ideation phase followed consisting of a series of cross-team workshops for the teachers to share knowledge and ideas on the use of Paro with different 
children for different purposes. Based on short videos and small narratives derived from the data obtained in phase 1 they collaborated on developing didactic designs, i.e. different types of use scenarios (e.g. type of practice and its context), use applications (e.g. type of interaction and the role of Paro) and user personas (e.g. student's individual needs, preferences and communication style), which could then be compared across teams. The teachers in T1 defined the goals for the use of Paro in their team at this stage, i.e. increasing bodily awareness through sensory stimulation and maintaining or shifting focus in certain crucial situations.

3. In the final co-creation phase the concepts of use were applied to systematize the implementation process in connection to the goals defined. At this point the teachers in T1 and T3 themselves restricted the use of Paro to 3-4 students, whom they considered were gaining the most from interacting with Paro. T2 made no restrictions on the use at any stage. During this phase we did follow-up visits at approximately one-weekly intervals, and the study was concluded with a focus group interview with all teachers involved in the final phase.

\section{$4 \quad$ Results and Discussion}

Results showed that the stage at which goal-setting activities were initiated did have an impact on participation (more teachers participated in T1 and T3 when compared to T2), motivation (the use of Paro was more frequent in T1 and T3 even though they had made restrictions on use and user personas, whereas $\mathrm{T} 2 \mathrm{had}$ not) and the potential for innovation (more didactic designs were developed in T1 and T3). When comparing the structured (T3) and semi-structured (T1) design processes, results indicate that although a structured design process can help ensure particularly ongoing participation and motivation, the semi-structured process seem to facilitate more reflection and thus innovation of the pedagogic practice, possibly since T1 had not decided on specific didactic designs prior to phase 2 and thus were more open to suggestions from the other teams. In addition to the results on participation, motivation and innovation we identified the four categories of contextual factors (knowledge, relations, values and flow) that affected the way in which Paro was implemented and used in practice. Thus these contextual factors should be considered when designing educational service robots for real-world educational settings.

\subsection{Knowledge}

In the case study we found, that the TSS framework enabled us to gain insight into how the technological intervention affects and is affected by the embedded knowledge in the environment and its traditions for sharing knowledge. For instance, one teacher's reflections on a workshop session show the opportunity that TSS brings for inspiration across teams: "It was a really good idea to try and use Paro as a companion during the mandatory psychological tests as one other teacher suggested". Another teacher reflected upon the time management system in the environment and how it did or did not support the introduction of the robot: "So far my sessions with Paro have been 
introductory with sequences of five minutes. Maybe ten minutes. Actually it is difficult to estimate the time. It's actually a little strange that I lose track of time, since we keep very strict time schedules". A third teacher explained how he had gained knowledge on the application of new technology in his context of use: "We should not replace existing support systems that work. The point is to find areas where the child shows potential for development but where we somehow lack the tools". These results show that the framework provided a platform for the participants to both share and create new knowledge by reflecting on their own practice. Thus, the framework not only provides researchers with knowledge about practice, it provides the opportunity to develop, share and use knowledge in collaboration with practice.

\subsection{Social Relations}

Experimental r-learning often focuses on the social relations between child and robot. However, in the case study we found that other social relations within the context can be of equal importance, i.e. child/child, child/teacher, teacher/robot or teacher/teacher relations. In some cases the social relation between a teacher and a child alone ensured a successful intervention with Paro: "We brought Paro along tobogganing and the student gave Paro a ride on the sledge. It has been several years since we were able to get him to go tobogganing with us. Of course, it was also a little stressful for him, but he enjoyed it. It's also kind of a special case because I've known him for many years and I am able to see that he is experiencing something positive, even though outside viewers may think he's a little stressed out". Here the teacher's relationship with the child enables her to explore the potential for Paro to motivate otherwise rejected opportunities for play. In yet other situations the social relationship itself was the actual goal of the intervention. For instance, in one case Paro was used as a social mediator between two children of which one was afraid of the other: "Paro has been an object of joint attention. During the sessions with Paro they have been able to approach each other while petting and grooming Paro. At one point they also briefly touched each other". In another case, the goal was for a student to be able to approach new people: "We brought Paro when visiting the school where a student will be going next year. She introduced Paro to the other students and was more confident than usual". These examples illustrate ways in which existing social relations can mediate the use of technology and affect the outcome of the intervention.

\subsection{Values}

From the case study we found that underlying values, and particularly competing values, in the educational environment mediated both the teachers' attitudes towards the robot as well as new technology in general. For instance, issues related to both attending students' individual needs and meeting outside academic standards was a source of frustration for some teachers: "It doesn't really make sense that the parents have to apply for dispensation from the common national academic tests when these students' level of cognitive development is estimated equivalent to 0-1 years of age". For other teachers, this conflict of values caused an increased awareness of one's own 
professionalism and reflections on whether the teacher should promote independency or intentionality in daily activities, e.g. by continuously prompting culturally defined "meaningful" play as opposed to letting the children play on their own in a perhaps to outside viewers less meaningful way. This shows that not only the attitudes of direct users and the values in the immediate context of the intervention are of importance when implementing robots in educational environments. Also indirect and potential users, parents and policymakers represent a group of stakeholders whose views and values affect and are affected by the implementation of educational service robots.

\subsection{Flow}

Here the term flow has several meanings. Firstly, the TSS framework provided the opportunity to gain insight into the daily flow of people in the context which is very much related to the above mentioned identification of indirect users and stakeholders. For instance, some children were living with their parents whereas others were permanently placed in care, visiting their parents once a week. Secondly, it emphasized the advantages of true long-term interaction in comparison with serial short-term interactions. The accessibility to the technology in the case study created opportunities for both consistency and flexibility in the r-learning designs and thus flow in the interaction. For instance, one teacher was able to apply Paro at certain crucial time to create and sustain flow: "We were at the church for Christmas. One student was feeling uneasy, covering his ears and repeating "close the door, close the door". Luckily I had brought Paro, so I showed it to him and said "look who it is" and he took down his hands to touch Paro and smiled". Here, the teacher's experience made it possible to exploit an optimal moment for intervention. Such moments occur spontaneously and cannot be orchestrated. Thus they are much less likely to occur in experimental settings where time and space are completely elided in favor of structure.

\section{Conclusions, Limitations and Directions for Future Research}

This paper presents work related to the development of a participatory design framework for involving users and practitioners in robot-supported didactic design processes, arguing a balanced level of time, space and structure e.g. by using goalsetting strategically at certain stages in the process. The applicability of the framework to real-world educational environments was demonstrated through a case study in robot-supported didactic designs for children with autism which revealed knowledge, social relations, values and flow as important factors when developing didactic designs with and for users and practitioners in autism education. The case study focuses on special needs education and the results are thus limited to this particular educational context. For the framework to be applicable to different educational contexts, e.g. specific domain didactics it must be challenged and further developed in different educational contexts, using different robotic platforms for different educational purposes. 


\section{References}

1. E.g. Institute for Personal Robots in Education, http: / / www . roboteducation.org/

2. Han, J.: Robot-Aided Learning and r-Learning Services. In: Chugo, D. (ed.) Human-Robot Interaction. InTech (2010), doi:10.5772/8143, ISBN: 978-953-307-051-3

3. Belpaeme, T., et al.: Multimodal Child-Robot Interaction: Building Social Bonds. Journal of Human-Robot Interaction 1(2), 33-53 (2012), http: / / www . aliz-e.org

4. Mataric, M.J., Koenig, N., Feil-Seifer, D.: Materials for enabling hands-on robotics and STEM education. AAAI Spring Symposium, Resources for AI Education (2007)

5. Majgaard, G., Nielsen, J., Misfeldt, M.: Robot Technology and Numbers in the Classroom. In: CELDA 2010, International Association for Development, pp. 231-234 (2010)

6. Han, J., Dongho, K.: r-Learning services for elementary school students with a teaching assistant robot. In: 4th International Conference on Human-Robot Interaction (2009)

7. Diehl, J.J., Schmitt, L.M., Villano, M., Crowell, C.R.: The Clinical Use of Robots for Individuals with Autism Spectrum Disorders: A Critical Review. Research in Autism Spectrum Disorders 6(1) (2012)

8. Lee, C.J., Kim, K., Breazeal, C., Picard, R.: Shybot: Friend-Stranger Interaction for Children Living with Autism. In: CHI 2008 (2008)

9. Robins, B., et al.: From Isolation to Communication: A Case Study Evaluation of Robot Assisted Play for Children with Autism with a Minimally Expressive Humanoid Robot. In: 2nd IEEE International Conference on Advances in Computer-Human Interactions (2009)

10. Kozima, H., Nakagawa, C., Yasuda, Y.: Interactive Robots for Communication-care: A Case Study in Autism Therapy. In: IEEE International Workshop on Robot and Human Interactive Communication, ROMAN 2005 (2005)

11. Hendler, J.: Robots for the rest of us: designing systems "out of the box", Robots for Kids: Exploring new technologies for learning. The Morgan Kaufmann Publishers (2000)

12. Goodrich, M.A., Schultz, A.C.: Human-Robot Interaction: a survey. Foundations and Trends in Human-Computer Interaction 1(3) (2007)

13. Papert, S.: Mindstorms: Children, Computers, and Powerful Ideas. Basic Books (1980)

14. Bers, M.U., et al.: Teachers as Designers: Integrating Robotics in Early Childhood Education. Information Technology in Childhood Education Annual (2002)

15. Hamner, E., et al.: Robot Diaries: Broadening Participation in the Computer Science Pipelin through Social Technical Exploration. In: AAAI Spring Symposium on Using AI to Motivate Greater Participation in Computer Science (2008)

16. Salter, T., Werry, I., Michaud, F.: Going Into the Wild in Child-Robot Interaction Studies: Issues in Social Robotic Development. Intelligent Service Robots 1(2) (2008)

17. Riek, L.D.: Wizard of Oz Studies in HRI: A Systematic Review and New Reporting Guidelines. Journal of Human-Robot Interaction 1(1), 119-136 (2012)

18. Leite, I., et al.: Long-term Interaction with Empathic Robots: Evaluating Perceived Support in Children. International Conference on Social Robotics (2012)

19. Shimada, M., Kanda, T., Koizumi, S.: How Can a Social Robot Facilitate Children's Collaboration? International Conference on Social Robotics (2012)

20. Von Hippel, E.: Democratizing Innovation: The Evolving Phenomenon of User Innovation. International Journal of Innovation Science 1(1), 29-40 (2009) 